Zawidzki M., Geometrical Universality of Truss-Z System .

In: Cocchiarella L. (eds) ICGG 2018 - Proceedings of the 18th

International Conference on Geometry and Graphics. ICGG 2018.

Advances in Intelligent Systems and Computing, vol 809. Springer, Cham, 2019.

\title{
Geometrical Universality of Truss-Z System
}

\author{
Machi Zawidzki \\ Institute of Fundamental Technological Research, Polish Academy of Sciences \\ Warsaw, Poland \\ zawidzki@mit.edu
}

\begin{abstract}
Standardization and modularization are common means of simplification and economization of engineering structures. Extremely modular systems (EMS) are comprised of very few (ideally just one) types of modules and allow for creation of structurally sound free-form constructions. Truss-Z (TZ) is the EMS considered in this paper. It is a skeletal system for creating free-form pedestrian ramps and ramp networks among any number of terminals in space. TZ structures are composed of four variations of a single basic unit subjected to affine transformations (mirror reflection, rotation and their combination). A family of shapes including: isosceles quadrilaterals (concave and convex), regular and irregular kites and darts, isosceles triangles and trapezoids has been considered for the planar projection of Truss-Z modules (TZMs). It has been shown that isosceles triangles and trapezoids suit best TZ. The universality of TZM has been assessed by measurement of the regularity of distribution reachable points by given TZ built with several such TZMs. It has been shown that the vertex angle $(\theta)$ of $32.5^{\circ}$ gives the most regularly distributed, thus universal $\mathrm{TZ}$.
\end{abstract}

Keywords: Truss-Z, Extremely Modular System, Isosceles Quadrilateral, Regularity.

\section{Introduction}

Standardization and modularization are common means of simplification and economization of engineering structures. Extremely Modular Systems are comprised of very few (ideally just one) types of modules and allow for creation of structurally sound free-form constructions.

\subsection{What is an Extremely Modular System?}

The concept of Extremely Modular System (EMS for short) has been introduced in [1]. In a nutshell, the purpose of EMS is to create free-form modular objects in given environments without obstacle violations, and self-collisions. EMS jointly meets the following three criteria:

1. EMS allows for creation of structurally sound free-form structures.

2. The number of module types in EMS is minimal.

3. EMS is not derived from a regular tessellation of space. 
The fundamental property of EMS for design is that the EMS modules can be assembled in more than one way. The concepts of two EMSs: Truss-Z (TZ) and Pipe-Z (PZ) have been presented in References [2] and [3], respectively. Any two Pipe-Z modules can be connected at practically any relative twist angle, and any two Truss- $Z$ modules in a single-branch structure can be connected in four distinct configurations.

$\mathrm{PZ}$ is more fundamental, as it forms single-branch mathematical knots with a parametric module subjected to relative twist. Since no direction is distinguished in PZ, it is considered as a "fully $3 \mathrm{D}$ " system. PZ has been proposed as a deployable construction system e.g.: for space habitats and emergency connectors [4]. TZ is a skeletal system for creating free-form pedestrian ramps and ramp networks among any number of terminals in space, as shown in Fig. 1.1.
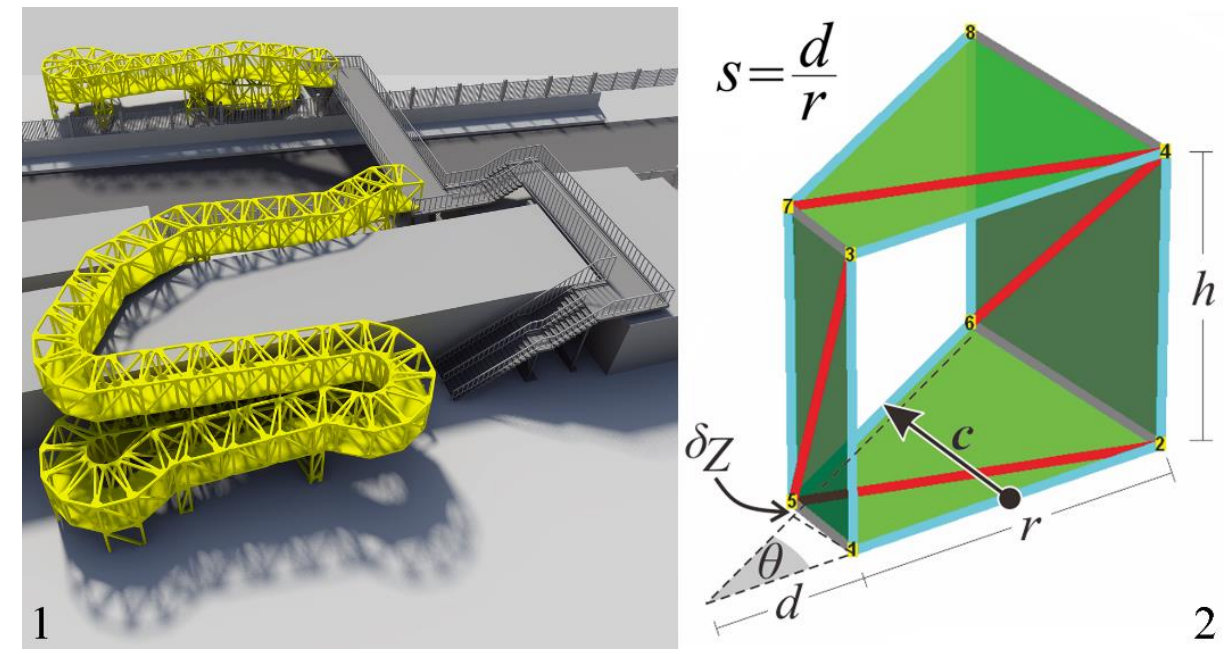

Fig. 1. On the left: a visualization of retrofitting of an existing overpass with Truss- $Z$ ramp for improved comfort and accessibility. On the right: the geometrical parameters of TZM. The "main frames", diagonal members and center-line vector $c$ are shown in cyan, red and black, respectively.

Since human movement along vertical $(Z)$ direction is different than in the horizontal $(\mathrm{XY})$ plane, TZ is considered as a " $2.5 \mathrm{D}$ " system. TZ structures are composed of four variations of a single basic unit subjected to the following affine transformations: mirror reflection, rotation and their combination. This gives effectively four "variations" of the basic module $R$ (which stands for "right", as according to the right-handrule it turns left and goes up), $R_{2}$ (rotated $R$ ), $L$ (which stands for "left", i.e. it is the mirror reflection of $R$ ) and $L_{2}$ (rotated $L$ ). As a result, there are $4^{n}$ possible assemblies of $n$ TZMs. Figure 2 shows all essentially unique TZ structures of length 3 , that is that is configurations unique up to the left-right and entrance-exit structural symmetries, as well as to their superposition (rotation). Thus, out of total $4^{3}=64$, there are 16 such essentially unique configurations. For detailed explanation see [5]. 


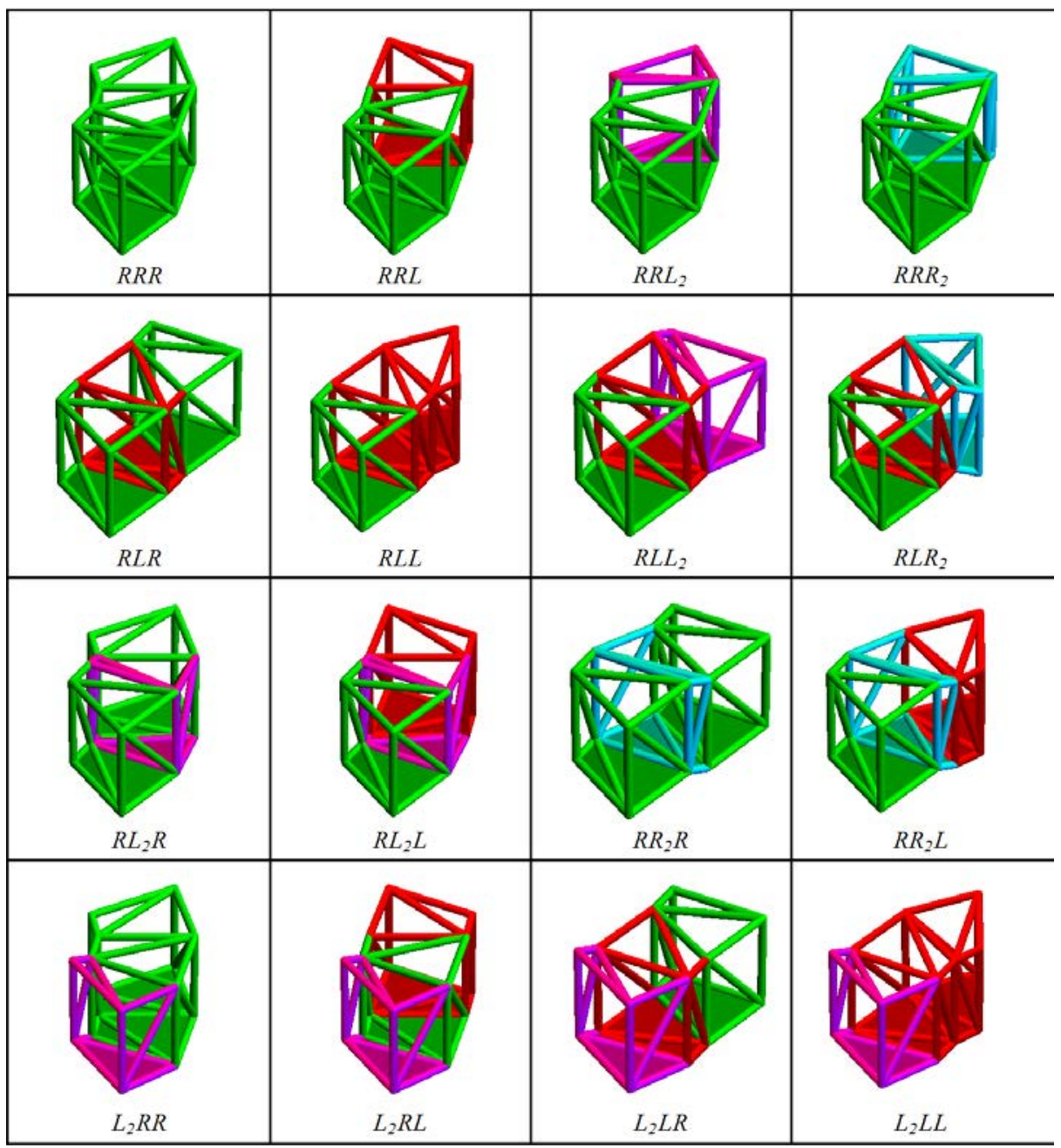

Fig. 2. 16 essentially unique configurations of three TZMs. The color convention is: $R, R_{2}, L$ and $L_{2}$ are shown in green, cyan, red and magenta, respectively.

Besides modularity, the second main quality of EMSs is universality. It can be expressed as the capability of "reaching any desired point of given space". Depending on the module parameters, a corresponding system can explore given space more or less efficiently. 


\section{Why the Truss- $\mathrm{Z}$ module is trapezoidal in the $\mathrm{XY}$ plane?}

The Truss- $Z$ module (TZM) is a frame-truss hybrid. The geometry of TZM is determined by the parameters: planar angle $\theta$, width $r$, "slenderness" $s$, vertical displacement $\delta_{Z}$, and height $h$, as shown in Figure 1.2.

The purpose of TZ is to create a path in $3 \mathrm{D}$ space which is structurally sound. To a certain degree, creation of a TZ structure can be considered as tiling of such a path with TZMs. The original concept of $\mathrm{TZ}$ was meant as a ramp system for human use, thus the properties of TZM in horizontal and vertical planes are considered separately.

\subsection{Tiling of TZMs in the horizontal plane}

In the horizontal plane constructing of TZ is analogous to a path-tiling. Since any quadrilateral tessellates [6], it is straightforward to create a relatively straight path with it, as shown in Figure 2.

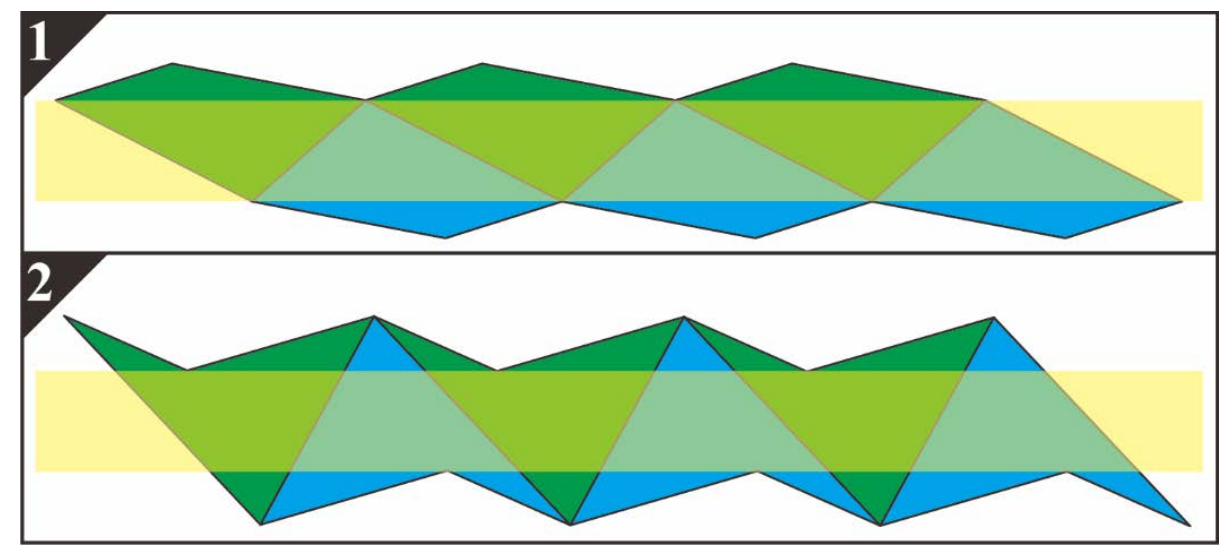

Fig. 3. Straight paths with the most general cases of irregular quadrilaterals, that is with four different angles and side lengths. A convex and concave quadrilateral are shown on the top and the bottom respectively. The basic tile and its rotation by $\pi$ are shown in green and blue, respectively. Yellow highlights the straight path of constant width.

It is important to note, that tiling requires nodes connect with nodes only. This means that a node of one tile cannot lie on an edge of another tile. For a brief discussion on regular (Platonic) tessellations occurring in Nature and used in engineering see [7].

However, with such quadrilaterals, it is not possible to make a "controlled turn", that is a turn with defined incremental angles.

Lemma 1: In order to perform this type of a path-tiling, a quadrilateral tile must meet two criteria:

1. two of its sides must have equal lengths (must be congruent), and

2. they must not be parallel. 
Congruent sides are called legs. Depending on the location of the legs, there are four types of such quadrilaterals: concave and convex isosceles quadrilateral (IQc \& IQx for short), irregular kite and dart (IK \& ID for short), as illustrated in Figure 3.

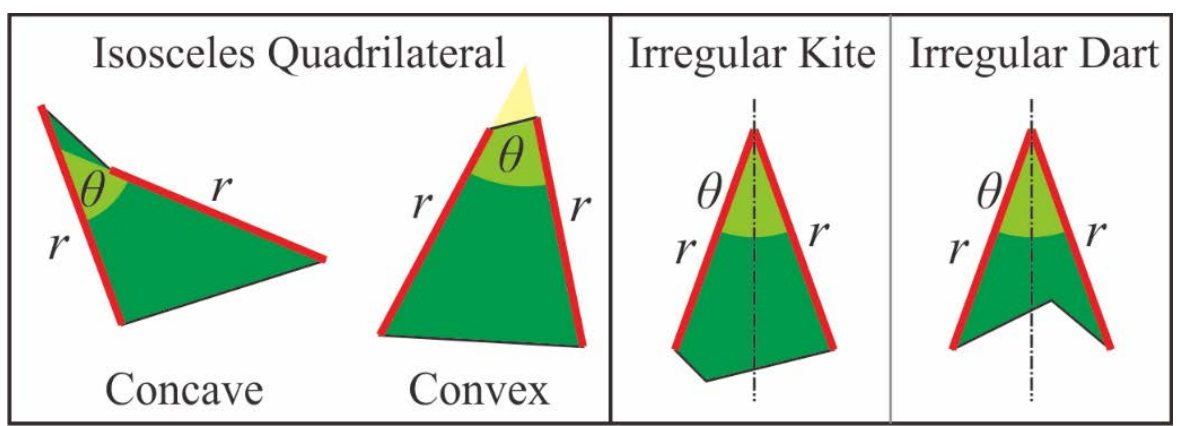

Fig. 4. From the left: two types of Isosceles quadrilaterals: concave $\left(\mathrm{IQ}_{\mathrm{c}}\right)$ and convex $\left(\mathrm{IQ}_{\mathrm{x}}\right)$, irregular kite (IK) and dart (ID). Congruent sides (legs) are highlighted in red.

Figure 4 below shows examples of straight and circular paths composed of the quadrilaterals shown in Figure 3.

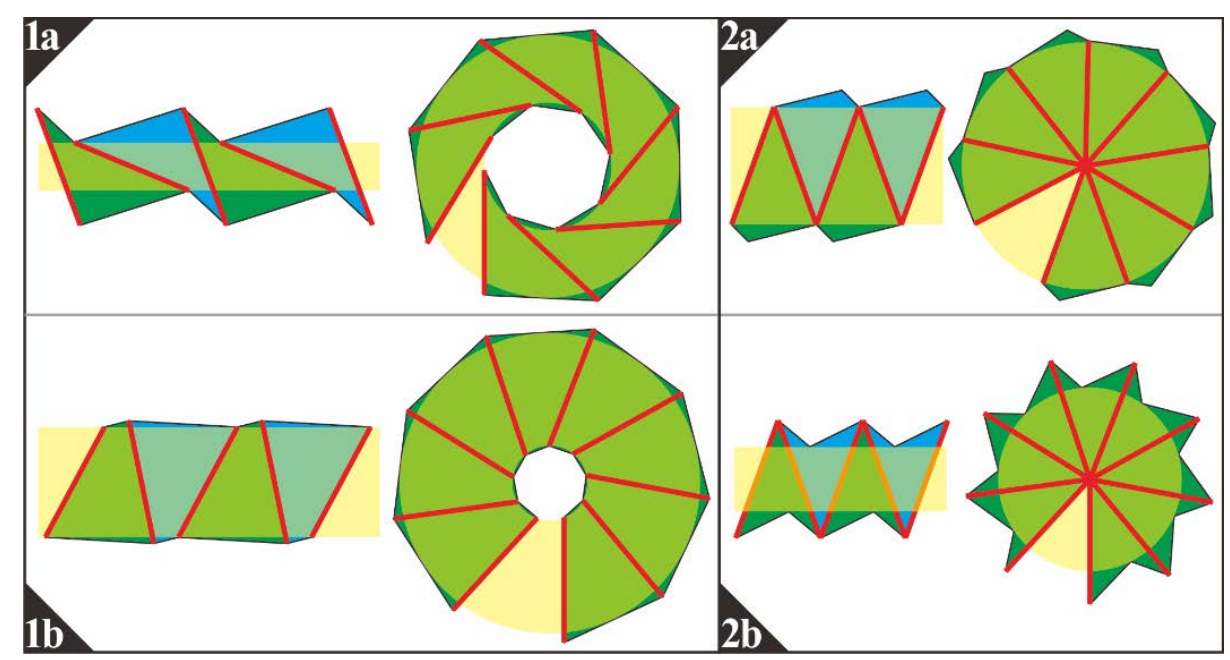

Fig. 5. Sub-figures $1 \mathrm{a}$ and $1 \mathrm{~b}$ show straight and circular paths composed of concave and convex IQs, respectively. Sub-figures $2 \mathrm{a}$ and $2 \mathrm{~b}$ show paths composed of irregular kites and darts, respectively. The widths of the paths vary for the straight and circular configurations. Legs are highlighted in red; otherwise, the color convention as in Figure 2.

IQ has the following properties:

1. IQ is a quadrilateral with one pair of opposite sides (legs) of equal length ( $r$ ).

2. The angle between the legs is denoted as $\theta, \theta:[0, \pi)$. 
3. Three degenerated cases of IQs:

i. If two pairs of opposite sides are congruent, IQ converges to a parallelogram. Obviously, if in addition all legs have the same length such IQ becomes a rhombus.

ii. If $\theta=0$, IQ converges to a rectangle. Obviously, if additionally, all sides are congruent, such an IQ becomes a square.

iii. If length of one of the sides other than leg is 0 , IQ degenerates to an isosceles triangle (which is not a quadrilateral anymore).

4. The special case of IQ: if legs are symmetrical, IQ becomes an isosceles trapezoid.

According to Lemma 1, neither parallelogram (and its special case, rhombus) or rectangle (and square) cannot serve as horizontal projections of a TZM. Kite and dart are acceptable TZM projections, however the more practical are: isosceles triangle and trapezoid, as illustrated in Figure 5.

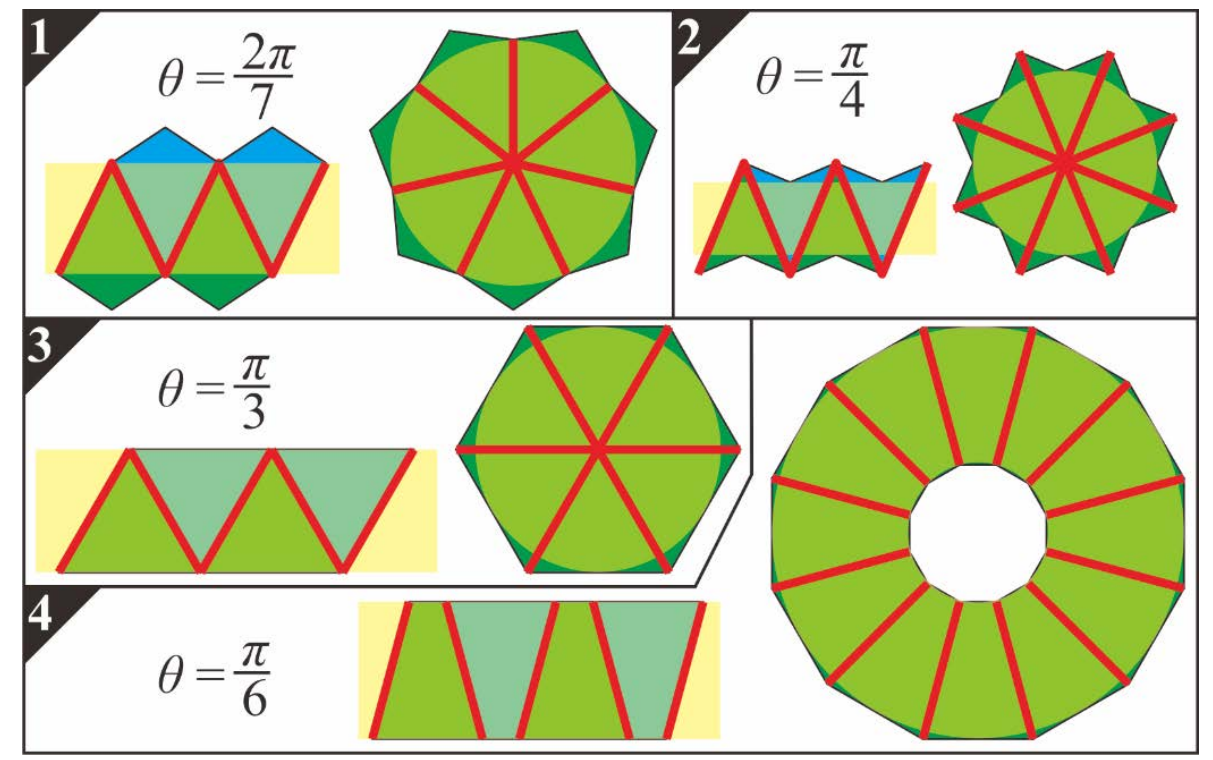

Fig. 6. Straight and circular paths composed of various cases of IQs. Sub-figures $1 \& 2$ show: kite and dart paths, respectively. Sub-figures $3 \& 4$ show paths composed of isosceles triangles and trapezoids, respectively. The values of angle $\theta$ are shown for each IQ. In all cases the path width is the same for straight and circular configuration.

Interestingly, IQ cannot become any other type of trapezoid than specifically the isosceles trapezoid. The angle $\theta$ can have any value from the interval $[0, \pi)$. However, restricting it to $2 \pi / k$, where $k$ is an integer greater than 2 , allows for creating closed loops where the first and last modules are aligned, as shown in Figure 5. Depending on the particular design, such condition may or may not be required. 
Lemma 2: the symmetry of such tile maximizes the effective width of straight and circular paths. Thus, isosceles triangle and trapezoid are the most suitable for the horizontal projections of TZM.

\subsection{Branching}

Ability of creating branching structures without necessity of additional special elements is also a desired feature for TZ. Since all quadrilaterals tessellate, in general sense, they can also branch. However, branching is understood here as ability of connecting multiple paths rather than ability of creating homogenous spreading structure. This is an arbitrary decision based on real-life case studies [8,9,10,11]. The reason for such approach is that the objective of TZ is to link two (or more) given terminals without violating obstacles. The number of terminals is usually not high, and the main challenge is the obstacle avoidance. Figures $6 \& 7$ show examples of branching in path-tiling with various IQs.

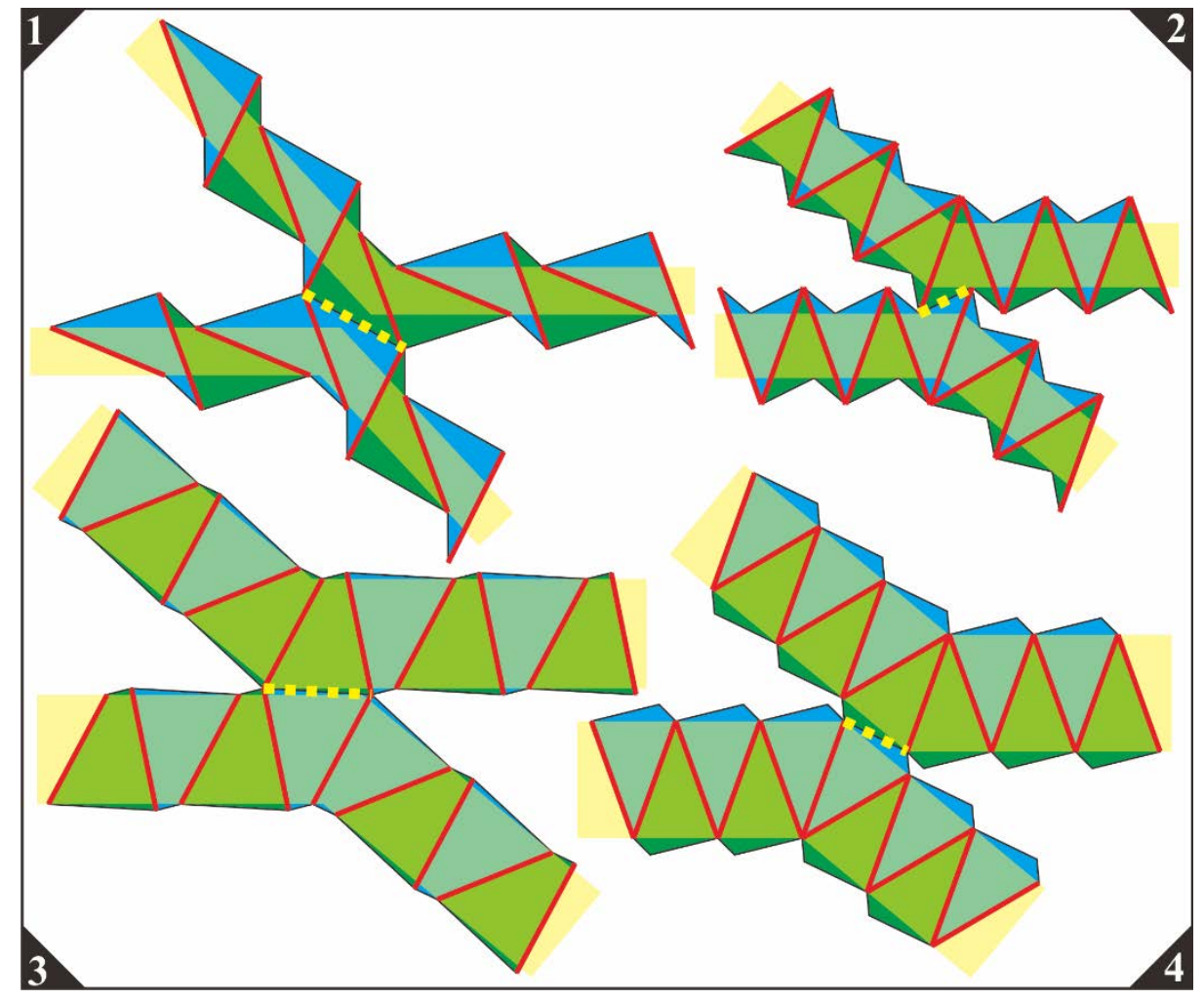

Fig. 7. Examples of branching. Sub-figures $1,2,3$, and 4 show configurations of: IQc, ID, IQx, and IK, respectively. Thick yellow dashing indicates the connection between the branches. It is also desirable that he width of such connection is similar to the width of the path through the tiling. 


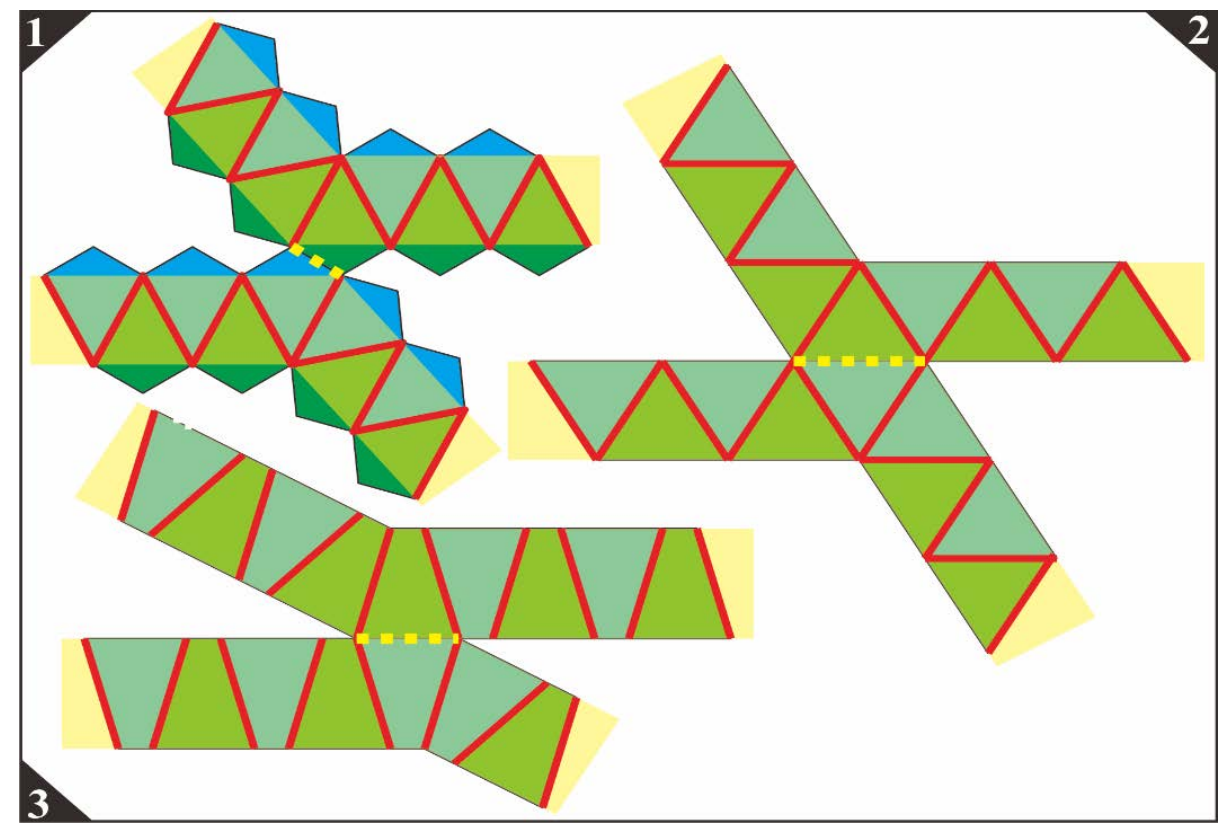

Fig. 8. Examples of branching continued. Sub-figures 1,2, and 3 show configurations of: kite, isosceles triangle and trapezoid.

\section{The measurement of universality of a single-branch TZ}

A measurement of TZ universality can be based on statistical evaluation of spatial distribution of points which are reachable by a structure assembled with a candidate TZM. In this method (presented independently in [12] and [13]), clustering, randomness and regularity form a continuum, described in terms of regularity $\mathbf{R}$.

$$
\mathbf{R}=\frac{2 \sum_{i \neq j}^{n} \min \left\{u_{i j}\right\}}{\sqrt{n A}}
$$

where $n$ is the number of points, $u_{i j}$ is the distance between $i^{\text {th }}$ point and $j^{\text {th }}$ point, $A$ is the area.

If all points are superimposed, $\mathbf{R}=0$ which corresponds to extreme clustering. At random dispersion $\mathbf{R}=1$. Uniform distribution on square grid gives $\mathbf{R}=2$, and for triangular lattice arrangement $\mathbf{R}=2.149$. For detailed explanation see [14].

Figure 8 shows examples of TZs composed of 10 TZMs with different angles $\theta$ from $20^{\circ}$ to $45^{\circ}$. Since the projection of TZ on XY plane is considered, there are $2^{10}=1024$ such configurations. In this case the value of parameter $r$ is irrelevant because the areas are relative to the maximal length of a straight TZ. Also slenderness $s$ is not relevant as TZs are represented here by the midpoints between nodes 5 and 6 of each TZM (see Figure 1.2), i.e. here isosceles triangles and trapezoids are equivalent. 


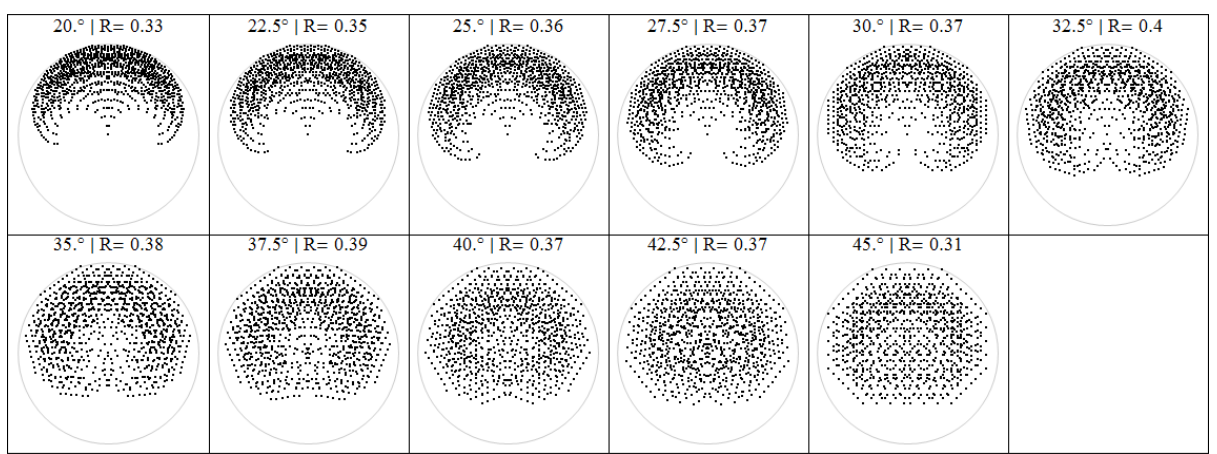

Fig. 9. Distribution on XY plane of all possible TZ paths constructed with 10 modules.

Many points in the Figure 9 are overlapping or very clustered. In order to improve clarity, Figure 10 shows these points "binned".

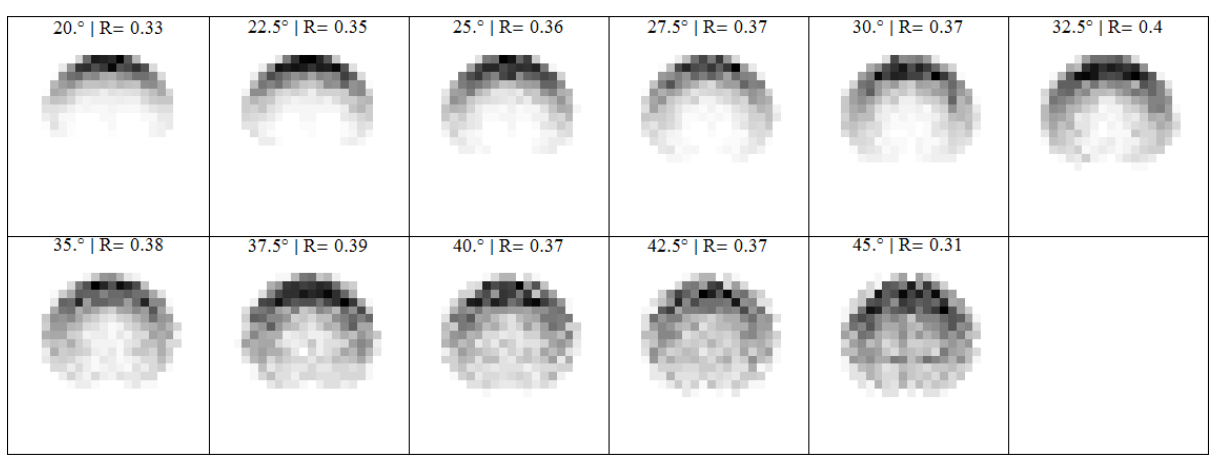

Fig. 10. The "heat map" of the points shown in Figure 9.

As Figure 9 and 10 indicate, the maximal regularity is reached at $\theta=32.5^{\circ}$

\section{Conclusions}

This paper presents the first attempt for systematical approach to establishing the "objectively" optimal parameters of Truss-Z module, which theretofore have been based on the designer's intuition of the author.

\section{Acknowledgments}

This work was completed as part of the project titled: "Innovative Extremely Modular Systems for temporary and permanent deployable structures and habitats: development, modeling, evaluation \& optimization". It was funded by "Polonez 2" research grant no. 2016/21/P/ST8/03856 supported by the National Science Centre, Poland. 
This project has received funding from the European Union's Horizon 2020 research and innovation program under the Marie Sklodowska-Curie grant agreement No 665778

\section{References}

1. Zawidzki M., Discrete Optimization in Architecture - Extremely Modular Systems, SpringerBriefs in Architectural Design and Technology, Springer Singapore, (2016).

2. Zawidzki M., Creating organic three-dimensional structures for pedestrian traffic with reconfigurable modular Truss-Z system, International Journal of Design \& Nature and Ecodynamics, 8(1), 61-87 (2013).

3. Zawidzki M., Nishinari K., Modular Pipe-Z system for three-dimensional knots, Journal for Geometry and Graphics, 17(1), 81-87 (2013).

4. Zawidzki M., Deployable Pipe-Z, Acta Astronautica 127, 20-30 (2016).

5. Zawidzki M., Jankowski L., Optimization of modular Truss-Z by minimum-mass design under equivalent stress constraint. Smart Structures and Systems 21(5) (2018).

6. Bart A., Clair B., Math and the Art of M.C. Escher: Tessellations by Quadrilaterals, http://mathstat.slu.edu/escher/index.php/Tessellations_by_Polygons\#Tessellations_by_Qu adrilaterals, last accessed 2018/05/13.

7. Zawidzki M., Dynamic shading of a building envelope based on rotating polarized film system controlled by one-dimensional cellular automata in regular tessellations (triangular, square and hexagonal). Advanced Engineering Informatics 29(1), 87-100 (2015).

8. Zawidzki M., Nishinari K., Application of evolutionary algorithms for optimum layout of Truss-Z. Advances in Engineering Software 65, 43-59 (2013).

9. Zawidzki - Optimization of Multi-branch Truss-Z based on evolution strategy. Advances in Engineering Software 100, 113-125 (2016).

10. Zawidzki M., - Retrofitting of pedestrian overpass by Truss- $Z$ modular systems using graph-theory approach. Advances in Engineering Software 81, 41-49 (2015).

11. Zawidzki M., Szklarski J., Effective Multi-objective Discrete Optimization of Truss-Z Layouts Using a GPU, Applied Soft Computing (in print).

12. Clark, P. J., Evans, F. C., Distance to nearest neighbor as a measure of spatial relationships in populations. Ecology 35, 445-453 (1954).

13. Hertz, P., Uber den geigenseitigen durchschnittlichen Abstand von Punkten, die mit bekannter mittlerer Dichte im Raume angeordnet sind. Mathematische Annalen 67, 387398 (1909).

14. Dry, M, Preiss, K., Wagemans, J., Clustering, Randomness, and Regularity: Spatial Distributions and Human Performance on the Traveling Salesperson Problem and Minimum Spanning Tree Problem. The Journal of Problem Solving 4(1), 1-16 (2012). 